\title{
Hepatic Resection for Non-functional Neuroendocrine Liver Metastasis: Does the Presence of Unresected Primary Tumor or Extrahepatic Metastatic Disease Matter?
}

\author{
Jun-Xi Xiang, MD ${ }^{1}$, Xu-Feng Zhang, MD, PhD ${ }^{1}$, Eliza W. Beal, $\mathrm{MD}^{2}$, Matthew Weiss, $\mathrm{MD}^{3}$, Luca Aldrighetti, $\mathrm{MD}^{4}$, \\ George A. Poultsides, $\mathrm{MD}^{5}$, Todd W. Bauer, $\mathrm{MD}^{6}$, Ryan C. Fields, $\mathrm{MD}^{7}$, Shishir Kumar Maithel, $\mathrm{MD}^{8}$, \\ Hugo P. Marques, MD ${ }^{9}$, and Timothy M. Pawlik, MD, MPH, PhD, FACS, FRACS (Hon.) ${ }^{2,10}$ \\ ${ }^{1}$ Department of Hepatobiliary Surgery and Institute of Advanced Surgical Technology and Engineering, The First \\ Affiliated Hospital of Xi' an Jiaotong University, Xi' an, China; ${ }^{2}$ Department of Surgery, The Ohio State University Wexner \\ Medical Center, Columbus, OH; ${ }^{3}$ Department of Surgery, Johns Hopkins Hospital, Baltimore, MD; ${ }^{4}$ Department of \\ Surgery, Scientific Institute San Raffaele, Vita-Salute San Raffaele University, Milan, Italy; ${ }^{5}$ Department of Surgery, \\ Stanford University, Stanford, CA; ${ }^{6}$ Department of Surgery, University of Virginia, Charlottesville, VA; ${ }^{7}$ Department of \\ Surgery, Washington University, School of Medicine, St Louis, MO; ${ }^{8}$ Department of Surgery, Emory University, Atlanta, \\ GA; ${ }^{9}$ Department of Surgery, Curry Cabral Hospital, Lisbon, Portugal; ${ }^{10}$ Department of Surgery, The Shelly and Urban \\ Meyer Professor of Cancer Research, The Ohio State University, Columbus, $\mathrm{OH}$
}

\begin{abstract}
Objectives. The objective of this study was to assess the impact of unresected primary tumor, as well as extrahepatic metastasis, on the long-term prognosis of patients undergoing hepatic resection for non-functional neuroendocrine liver metastasis (NF-NELM).

Methods. Patients who underwent hepatic resection for NF-NELM were identified from a multi-institutional database. Data on clinical and pathological details, as well as the long-term overall survival (OS) were obtained and compared. Propensity score matching was performed to generate matched pairs of patients.
\end{abstract}

Results. Among the 332 patients with NF-NELM, 281 $(84.6 \%)$ underwent primary tumor resection, while 51

Electronic supplementary material The online version of this article (https://doi.org/10.1245/s10434-018-6751-8) contains supplementary material, which is available to authorized users.

Jun-Xi Xiang and Xu-Feng Zhang have contributed equally to the study.

(C) Society of Surgical Oncology 2018

First Received: 13 May 2018;

Published Online: 14 September 2018

T. M. Pawlik, MD, MPH, PhD, FACS, FRACS (Hon.)

e-mail: tim.pawlik@osumc.edu
(15.4\%) did not. Patients who underwent primary resection were more likely to have a pancreatic primary and metachronous NELM. The long-term OS of patients who did and did not have the primary neuroendocrine tumor (NET) resected was comparable on both unmatched (10-year survival rate $66.8 \%$ vs. $54.0 \%, p=0.192$ ) and matched (10-year survival rate $75.7 \%$ vs. $60.4 \%, p=0.271)$ analyses. In contrast, patients with NF-NELM and extrahepatic metastasis had a worse OS following resection compared with patients who had intrahepatic-only metastasis on unmatched (10-year survival rate $37.5 \%$ vs. $69.3 \%$, $p=0.002)$ and matched (10-year survival rate $37.5 \%$ vs. $86.3 \%, p=0.011)$ analyses. On multivariable analysis, while resection of the primary NET was not associated with OS (hazard ratio [HR] 0.7, 95\% confidence interval [CI] $0.4-1.2, p=0.195$ ), the presence of extrahepatic metastasis was independently associated with long-term risk of death (HR 3.9, 95\% CI 1.7-9.2, $p=0.002$ ).

Conclusions. While surgery should be considered for patients with NF-NELM who have an unresectable primary tumor, operative resection of NF-NELM may not be as beneficial in patients with extrahepatic disease.

Gastroenteropancreatic neuroendocrine tumors (GEPNETs) are a heterogeneous group of rare tumors arising mainly from the pancreas and gastrointestinal tract. ${ }^{1}$ While some GEP-NETs may have an indolent course, the liver is 
a common metastatic site for GEP-NETs. In fact, up to $60-90 \%$ of patients may develop synchronous or metachronous neuroendocrine liver metastasis (NELM) during the course of their disease.

Surgical resection is the main curative treatment option for patients with NELM. ${ }^{2}$ Treatment goals for NELM often include both prolongation of survival and alleviation of tumor-related symptoms, which can include debilitating hormonal symptoms and mechanical symptoms. ${ }^{3}$ While a subset of patients have functional tumors, a recent study of 9281 patients from the National Cancer Data Base with pancreatic NETs demonstrated that over $75 \%$ of NETs were non-functional. ${ }^{4}$ Due to the lack of early symptoms, non-functional neuroendocrine tumors with liver metastasis (NF-NELM) are often discovered late, which can result in a higher incidence of the primary lesion being unresectable and/or the presence of extrahepatic metastasis. ${ }^{4-6}$ NF-NETs are also more likely to be malignant and are often associated with worse outcomes versus functional NETs. ${ }^{6-9}$

Traditionally, the presence of unresectable primary disease and/or extrahepatic metastatic disease was considered advanced NELM. ${ }^{10}$ While some investigators have recommended against resection of NELM in the setting of advanced disease, ${ }^{11}$ other data have supported surgical debulking as a reasonable treatment of NELM that has yielded improved long-term survival. ${ }^{12}$ As most patients with NELM die of liver failure due to intrahepatic tumor progression rather than the primary tumor or extrahepatic disease, liver resection may have a role, even in the setting of advanced disease. ${ }^{12,13}$ However, whether the presence of an unresectable primary tumor and/or extrahepatic metastatic disease should be considered a contraindication to liver resection for NF-NELM remains debated. As such, the objective of the current study was to define the impact of an unresected primary tumor and/or extrahepatic metastatic disease on the long-term prognosis of patients undergoing hepatic resection for NF-NELM.

\section{METHODS}

\section{Study Cohort}

A total of 548 patients who underwent liver-directed therapy for NELM from January 1980 to December 2015 were identified from nine international institutions. The Institutional Review Board of all participating institutions approved the study. The diagnoses of all NELM patients were confirmed histologically. Patients with hormonally functional NETs $(n=201)$, receipt of liver ablation only $(n=11)$, and patients who died within 30 days after surgery $(n=4)$ were excluded.

\section{Data Collection and Follow-Up}

Data on clinical, operative, and pathological details were collected for each patient at each institution in a standardized database pertaining to both the primary tumor and liver metastases. Extrahepatic metastatic disease was determined by imaging studies, e.g. computed tomography (CT) scan, octreoscan, positron emission tomography (PET)-CT, etc., and/or biopsy before surgery among the different centers. Grade of tumor differentiation was classified as well (G0), moderate (G1), or poor (G3) according to the 2010 WHO grading system. ${ }^{14}$ An R0 resection was defined as the absence of macroscopic or microscopic disease at the surgical margin, while an $\mathrm{R} 1$ resection was defined as the microscopic presence of tumor and R2 was classified as macroscopic presence of tumor. ${ }^{15}$

\section{Statistical Analysis}

Continuous variables were expressed as medians with interquartile ranges (IQRs), while categorical variables were reported as totals and frequencies. Univariable comparisons were assessed by using the Mann-Whitney $U$ test, Chi square test, or Fisher exact test, as appropriate. Survival was evaluated using Kaplan-Meier curves and median values were compared using the log-rank tests. The impact of various clinicopathological factors on overall survival (OS) was assessed using a Cox proportional hazards model and expressed as hazard ratio (HR) and $95 \%$ confidence interval (CI). Factors with a $p$ value $<0.05$ by univariate analysis were included in the multivariate analysis. Propensity score matching (PSM) was used to adjust for differences in baseline characteristics between groups. In all analyses, a $p$ value $<0.05$ (two-tailed) was considered statistically significant. Statistical analyses were performed using SPSS 22.0 (IBM Corporation, Armonk, NY, USA).

\section{RESULTS}

\section{Demographic and Clinicopathological Characteristics}

Among the 548 patients diagnosed with GEP-NETs, a total of $332(60.6 \%)$ patients who underwent hepatectomy for NF-NELM were included in the analytic cohort (Table 1). Median patient age was 58 years (IQR 49-67) and nearly half of patients were female $(n=151,45.5 \%)$. Among all patients, $187(56.3 \%)$ presented with nonspecific clinical symptoms, including abdominal cramping. Primary tumor site included the pancreas $(n=149,44.9 \%)$, gastrointestinal tract $(n=129,38.8 \%)$, tracheobronchial and lung ( $n=14,4.2 \%)$, and unknown location $(n=40$, $12 \%)$. Liver metastases were synchronous in $217(65.4 \%)$ 
TABLE 1 Clinicopathological characteristics of patients who underwent hepatic resection for non-functional neuroendocrine liver metastasis

\begin{tabular}{|c|c|c|c|c|}
\hline & All patients $(n=332)$ & Primary resected $(n=281)$ & Primary unresected $(n=51)$ & $p$ Value \\
\hline Age, years (IQR) & $58(49-67)$ & $58(49-67)$ & $48(42-67)$ & 0.863 \\
\hline Sex, male/female & $181(54.5) / 151(45.5)$ & $160(56.9) / 121(43.1)$ & $21(41.2) / 30(58.8)$ & 0.038 \\
\hline Symptomatic & $187(56.3)$ & $164(58.4)$ & $23(45.1)$ & 0.079 \\
\hline Primary tumor location & & & & $<0.001$ \\
\hline Non-pancreatic & $183(55.1)$ & $137(48.8)$ & $46(90.2)$ & \\
\hline Pancreatic & $149(44.9)$ & $144(51.2)$ & $5(9.8)$ & \\
\hline Synchronous liver metastasis & $217(65.4)$ & $176(62.6)$ & $41(80.4)$ & 0.014 \\
\hline Bilateral liver metastasis & $181(54.5)$ & $157(55.9)$ & $24(47.1)$ & 0.245 \\
\hline Estimated liver involvement & & & & 0.662 \\
\hline$<50 \%$ & $58(17.5)$ & $48(17.1)$ & $10(19.6)$ & \\
\hline$\geq 50 \%$ & $274(82.5)$ & $233(82.9)$ & $41(80.4)$ & \\
\hline Tumor grade & & & & 0.015 \\
\hline Well-differentiated & $129(57.1)$ & $122(59.5)$ & $7(33.3)$ & \\
\hline Moderately differentiated & $53(23.5)$ & $48(23.4)$ & $5(23.8)$ & \\
\hline Poorly differentiated & $44(19.5)$ & $35(17.1)$ & $9(42.9)$ & \\
\hline $\mathrm{NA} / \mathrm{missing}$ & 106 & 76 & 30 & \\
\hline Extrahepatic disease & $37(11.1)$ & $30(10.7)$ & $7(13.7)$ & 0.524 \\
\hline Preoperative treatment & & & & 0.218 \\
\hline Octreotide & $22(6.6)$ & $20(7.1)$ & $2(3.9)$ & \\
\hline Chemotherapy & $27(8.1)$ & $20(7.1)$ & $7(13.7)$ & \\
\hline None & $283(85.2)$ & $241(85.8)$ & $42(82.4)$ & \\
\hline Intraoperative ablation & $76(22.9)$ & $69(24.6)$ & $7(13.7)$ & 0.090 \\
\hline Type of hepatectomy & & & & 0.019 \\
\hline Parenchymal-sparing resection & $205(61.7)$ & $181(64.4)$ & $24(47.1)$ & \\
\hline Major resection & $127(38.3)$ & $100(35.6)$ & $27(52.9)$ & \\
\hline Margin & & & & 0.030 \\
\hline $\mathrm{R} 0 / \mathrm{R} 1$ & $291(87.7)$ & $251(89.3)$ & $40(78.4)$ & \\
\hline $\mathrm{R} 2$ & $41(12.3)$ & $30(10.7)$ & $11(21.6)$ & \\
\hline Adjuvant therapy & $129(38.9)$ & $114(40.6)$ & $15(29.4)$ & 0.133 \\
\hline
\end{tabular}

Data are expressed as $n(\%)$ unless otherwise specified

$I Q R$ interquartile range, $N A$ not available

patients and metachronous in $115(34.6 \%)$ patients. Bilateral liver disease was present in the majority of patients $(n=181,54.5 \%)$ and most patients had an estimated $\geq 50 \% \quad$ liver involvement $\quad(n=440, \quad 79.4 \%)$. Extrahepatic metastatic disease was noted in 37 (11.1\%) patients at the time of liver surgery. Most patients $(n=283,85.2 \%)$ did not receive any additional treatment before hepatectomy, while $22(6.6 \%)$ and $27(8.1 \%)$ patients had received octreotide or chemotherapy, respectively. Most patients $(n=205,61.7 \%)$ underwent a parenchymal-sparing resection. During the operation, tumor ablation was concomitantly performed in $76(22.9 \%)$ patients. On final pathology, $291(87.7 \%)$ patients had a curative-intent resection (R0/R1), while 41 (12.3\%) patients had a macroscopically positive (R2) surgical margin. The majority of patients $(n=203,61.1 \%)$ did not receive any adjuvant treatment, whereas 129 (38.9\%) patients received postoperative octreotide or chemotherapy.

\section{Overall Survival (OS): Primary Resected Versus Unresected Non-functional Neuroendocrine Liver Metastasis (NF-NELM)}

Among the 332 patients with NF-NELM, the primary tumor was resected in 281 (84.6\%) patients; 51 (15.4\%) patients had an unresected primary tumor at the time of liver surgery for NELM (Fig. 1). The 1-, 3-, 5-, and 10-year survival of the entire cohort was 97.4, 89.5, 82.2, and $65.1 \%$, respectively. Patients who had a resected primary 
tumor were more likely to be male $(56.9 \%$ vs. $41.2 \%$, $p=0.038)$ and have a pancreatic NET $(51.2 \%$ vs. $9.8 \%$, $p<0.001)$ versus patients who had a primary NET that was not resected (Table 1). More patients with an unresected primary tumor presented with a synchronous liver metastasis compared with patients who had the primary NET resected $(80.4 \%$ vs. $62.6 \%, p=0.014)$. Although tumor burden was not different among patients with a resected versus unresected primary tumor $(\geq 50 \%$ liver involvement, $82.9 \%$ vs. $80.4 \%, p=0.662$ ), patients who had the primary tumor resected were more likely to undergo parenchymal-sparing hepatic resection $(64.4 \%$ vs. $47.1 \%, p=0.019$ ) and had a higher incidence of curativeintent resection (R0/R1, 89.3\% vs. 78.4\%, $p=0.030$ ) versus patients with an unresected primary tumor (Table 1). Patients with a primary resected tumor were more likely to have a well-differentiated tumor $(59.5 \%)$, while patients with a primary unresected tumor were more likely to have a poorly differentiated tumor $(42.9 \%$, $p=0.015)$. Lymph node metastasis was present in 105 (37.4\%) patients.

Patients who underwent primary NET resection had a comparable long-term OS versus patients who had an unresected NET primary (10-year survival rate, primary resected $66.8 \%$ vs. unresected $54.0 \%, p=0.192$ ) [Fig. 2a]. The propensity-matched cohort included 90 patients who did or did not have the primary NET resected (Electronic Supplementary Table 1). In the matched cohort, OS was equivalent among patients who had the primary NET resected versus patients who had an unresected primary NET (10-year survival rate, primary resected $75.7 \%$ vs. unresected 60.4\%, $p=0.271$ ) [Fig. 2b]. Of note, no differences in OS were identified among patients undergoing hepatic resection for NF-NELM among the different eras (1980-2000 vs. 2001-2010 vs. after 2011, $p=0.396$ ) [Electronic Supplementary Fig. 1].

\section{OS: Presence of Extrahepatic Metastatic Disease Versus Intrahepatic-Only Metastasis}

At the time of NF-NELM resection, extrahepatic metastatic disease was noted in $37(11.1 \%)$ patients (Fig. 1). Extrahepatic sites included the lungs $(n=11)$, peritoneum $(n=19)$, bone $(n=7)$, and other sites $(n=12)$; 9 patients had multiple sites of extrahepatic metastases. Compared with patients who had intrahepatic-only disease, patients with NF-NELM plus extrahepatic metastasis were more likely to have a pancreatic primary tumor (47.5\% vs. 24.3\%, $p=0.008$ ) [Electronic Supplementary Table 2]. Patients with extrahepatic metastatic disease had a worse OS versus patients with intrahepatic-only metastasis (10year survival rate, $69.3 \%$ vs. $37.5 \%, p=0.002$ ) [Fig. 3a]. After 1:2 PSM, the analytic cohort included 95 patients who had comparable demographic and clinicopathologic characteristics (Electronic Supplementary Table 2). In the matched cohort, the presence of extrahepatic metastatic disease remained associated with worse long-term outcome (10-year survival, extrahepatic metastatic disease $36.3 \%$ vs. intrahepatic-only disease $77.9 \%, p=0.019$ ) [Fig. 3b].

\section{Risk Factors Associated with OS of NF-NELM}

On univariate analysis, symptomatic, pancreatic primary NET, synchronous liver metastasis, R2 resection, tumor

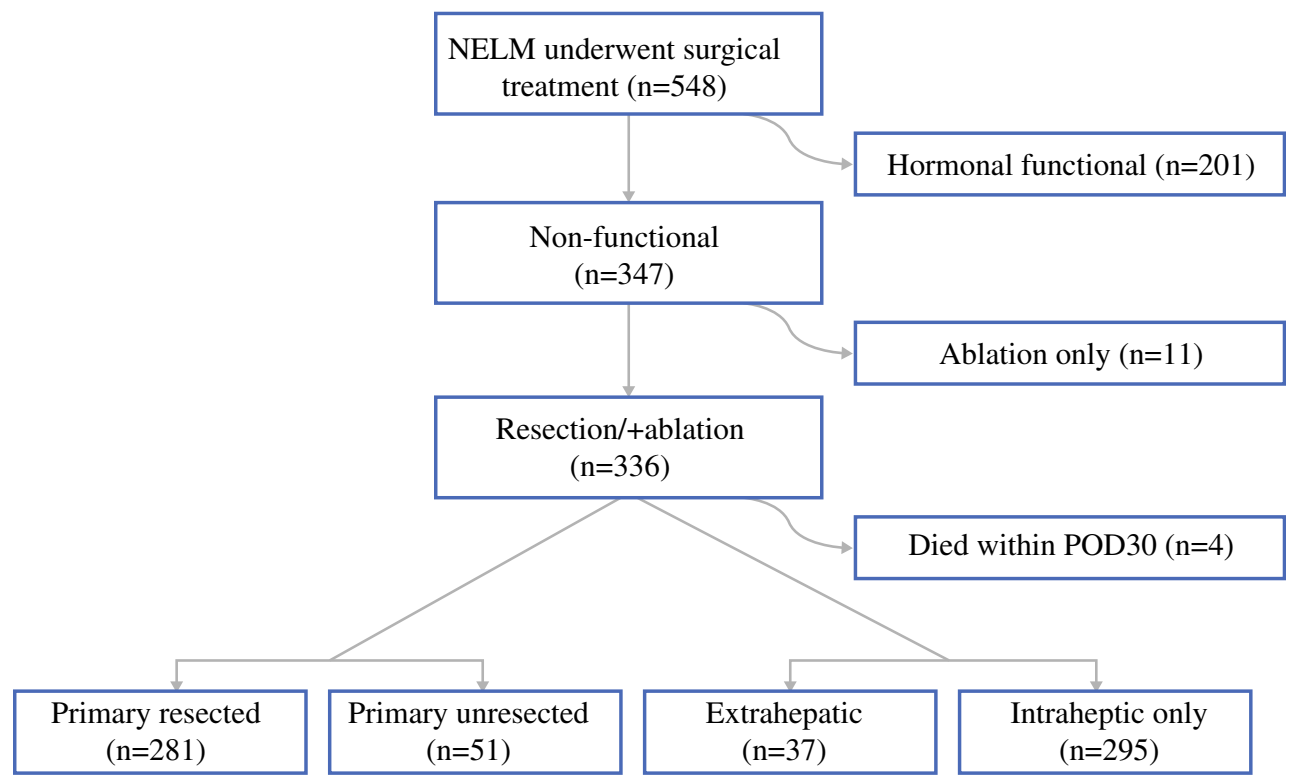

FIG. 1 Patient inclusion and study scenario. NELM neuroendocrine liver metastasis, $P O D$ postoperative day 


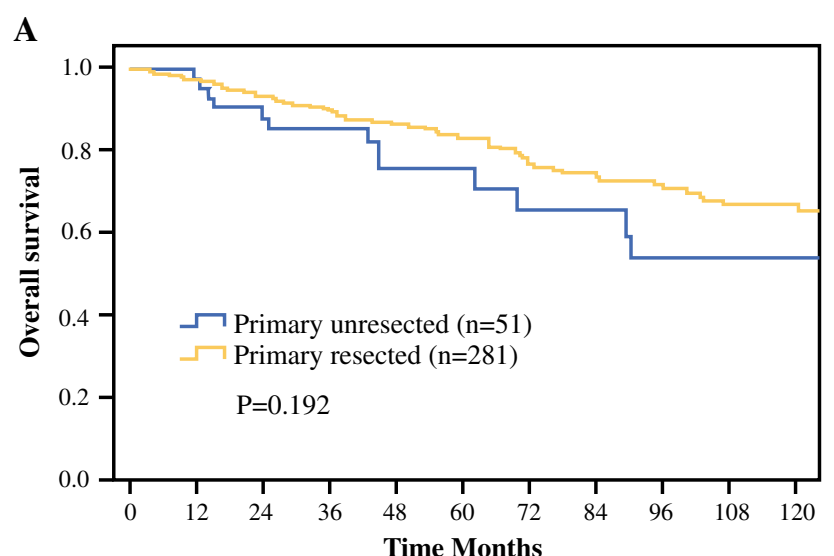

B

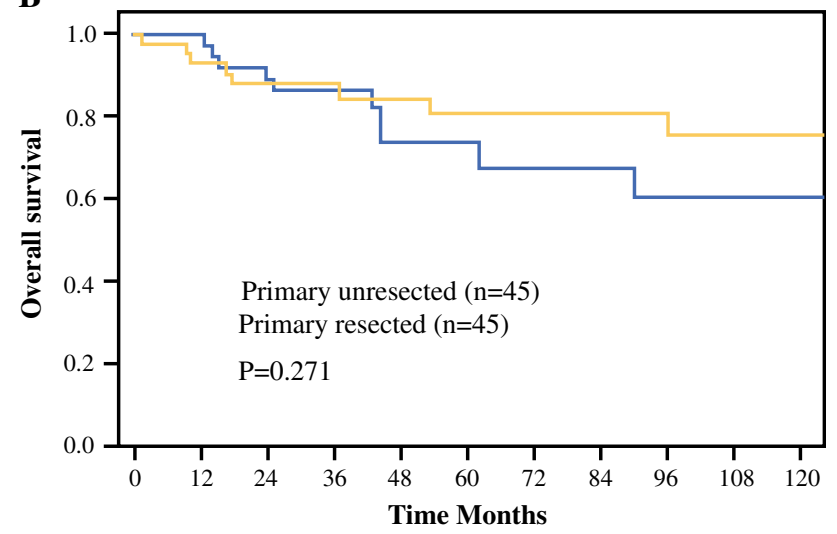

FIG. 2 Overall survival after curative-intent surgery for primary resected and unresected non-functional neuroendocrine liver metastasis before (a) and after (b) propensity score matching

grade, and extrahepatic metastatic disease were each associated with worse long-term survival among patients with NF-NELM (Table 2). Of note, resection of the primary NET was not associated with OS (HR 0.7, 95\% CI $0.4-1.2, p=0.195)$. On multivariable analysis, after taking into account all potential confounding factors, the presence of extrahepatic metastatic disease (HR 3.9, 95\% CI 1.7-9.2, $p=0.002$ ) remained independently associated with worse outcome among patients with NF-NELM.

\section{DISCUSSION}

With improvements in abdominal imaging, the incidence of NF-NETs has increased to $50-75 \%$ of all GEPNETs. ${ }^{16}$ In fact, over half of NF-NET patients are asymptomatic and have disease sporadically discovered on imaging for an unrelated problem. ${ }^{14,17,18}$ Due to occult progression and delayed diagnosis, more NF-NET patients may present with an unresectable primary tumor and extrahepatic metastasis than patients with functional NETs. ${ }^{14,17,18}$ In turn, the presence of unresectable primary NETs and/or extrahepatic metastasis are still considered a
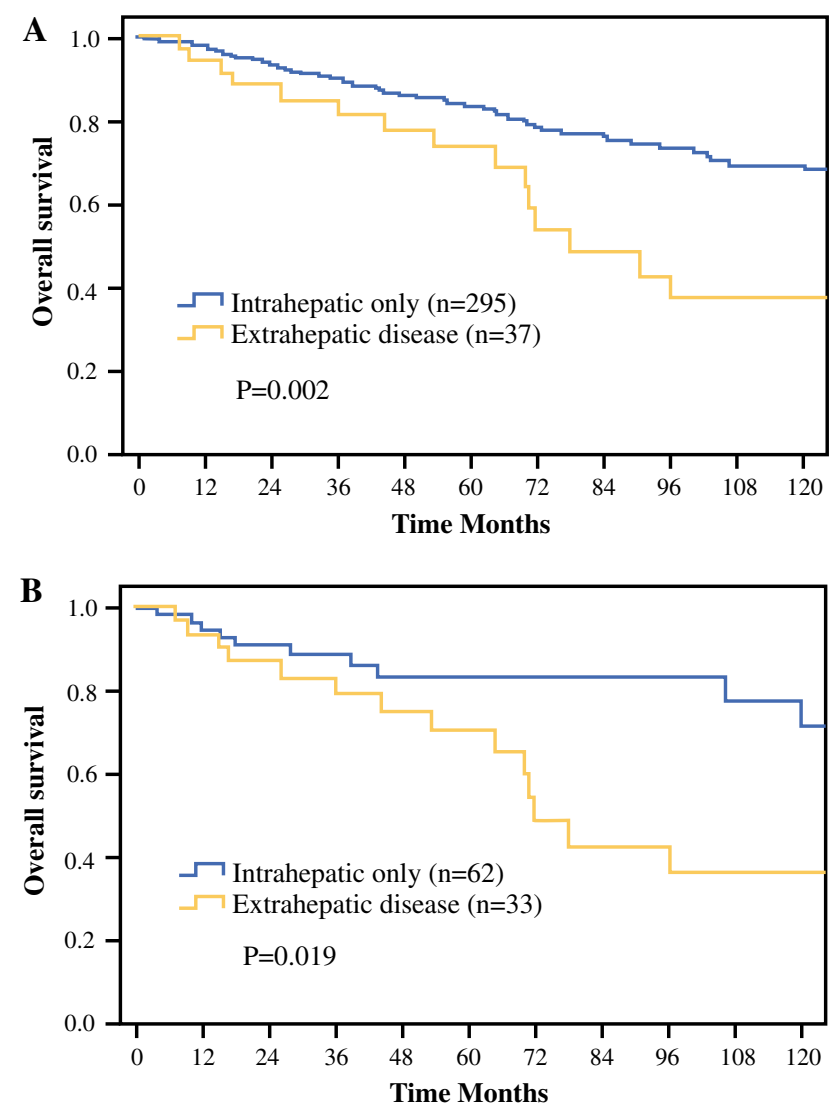

FIG. 3 Overall survival of non-functional neuroendocrine liver metastasis patients with or without extrahepatic metastatic disease before (a) and after (b) propensity score matching

contraindication to surgery by many clinicians. ${ }^{11,19}$ However, aggressive onco-surgery has been increasingly advocated, even in the presence of advanced metastatic disease. $^{20,21}$ The current study was important as it specifically defined the outcomes of patients with advanced NFNELM who had unresected primary NETs, as well as extrahepatic metastasis using a large, international, multiinstitutional database. Of note, long-term survival was comparable among patients who had the primary NET resected versus patients who had an unresectable primary NET left in situ. Comparable long-term OS among patients who did and did not have the primary NET resected was noted on both unadjusted (10-year survival rate $66.8 \%$ vs. $54.0 \%, p=0.192)$ and adjusted (10-year survival rate $75.7 \%$ vs. $60.4 \%, p=0.271$ ) analyses. In contrast, the presence of extrahepatic metastatic disease was strongly associated with a worse OS compared with intrahepaticonly disease as patients who had extrahepatic metastatic disease were noted to have a 2.5 -fold higher risk of death long-term.

The beneficial role of resecting the primary NET has been a topic of debate. Givi et al. reported on 84 patients with gastrointestinal carcinoid hepatic metastases and 
TABLE 2 Risk factors associated with overall survival rate of non-functional neuroendocrine liver metastasis

\begin{tabular}{|c|c|c|c|c|}
\hline & \multicolumn{2}{|c|}{ Univariate analysis } & \multicolumn{2}{|c|}{ Multivariate analysis } \\
\hline & $\mathrm{HR}(95 \% \mathrm{CI})$ & $p$ Value & $\operatorname{HR}(95 \% \mathrm{CI})$ & $p$ Value \\
\hline Age, $\leq 60 />60$ years & $0.9(0.6-1.4)$ & 0.613 & & \\
\hline Sex, male/female & $1.5(0.9-2.4)$ & 0.089 & & \\
\hline Symptomatic & $1.7(1.0-2.7)$ & 0.030 & $1.6(0.8-2.9)$ & 0.158 \\
\hline Primary tumor location & & 0.005 & & 0.002 \\
\hline Non-pancreatic & Ref. & & Ref. & \\
\hline Pancreatic & $1.9(1.2-3.1)$ & & $2.8(1.4-5.4)$ & \\
\hline Primary tumor resected & $0.7(0.4-1.2)$ & 0.195 & & \\
\hline Synchronous liver metastasis & $2.5(1.5-4.2)$ & $<0.001$ & $2.1(1.0-4.1)$ & 0.037 \\
\hline Bilateral liver metastasis & $0.9(0.6-1.5)$ & 0.758 & & \\
\hline Estimated liver involvement, $<50 \% / \geq 50 \%$ & $0.9(0.5-1.6)$ & 0.727 & & \\
\hline \multicolumn{5}{|l|}{ Tumor grade } \\
\hline Well-differentiated & Ref. & & Ref. & \\
\hline Moderately differentiated & $4.0(2.0-8.0)$ & $<0.001$ & $3.0(1.5-6.1)$ & 0.003 \\
\hline Poorly differentiated & $4.1(2.0-8.4)$ & $<0.001$ & $2.2(0.9-5.2)$ & 0.067 \\
\hline Extrahepatic disease & $2.4(1.3-4.1)$ & 0.002 & $3.9(1.7-9.2)$ & 0.002 \\
\hline \multicolumn{5}{|l|}{ Preoperative treatment } \\
\hline None & Ref. & & & \\
\hline Octreotide & $0.8(0.2-2.4)$ & 0.650 & & \\
\hline Chemotherapy & $1.7(0.8-3.6)$ & 0.197 & & \\
\hline Intraoperative ablation & $1.2(0.7-2.0)$ & 0.516 & & \\
\hline Types of hepatectomy & & 0.088 & & \\
\hline Parenchymal-sparing resection & Ref. & & & \\
\hline Major resection & $0.7(0.4-1.1)$ & & & \\
\hline Margin & & $<0.001$ & & 0.027 \\
\hline $\mathrm{R} 0 / \mathrm{R} 1$ & Ref. & & Ref. & \\
\hline $\mathrm{R} 2$ & $3.9(2.2-6.9)$ & & $2.5(1.1-5.6)$ & \\
\hline
\end{tabular}

$H R$ hazard ratio, $C I$ confidence interval, Ref. reference noted that primary tumor resection was associated with improved survival. ${ }^{22}$ However, all patients in this study received non-surgical treatment for NELM. ${ }^{22}$ Whether there is a role for surgical resection of NF-NELM in the setting of an unresected primary NET remains largely unknown. Importantly, data from the current study demonstrated comparable long-term survival among patients who did and did not have the primary NET resected. In fact, the presence of an unresected primary NET was not a risk factor for OS among patients undergoing resection of NF-NELM. As such, hepatic resection of NF-NELM should be strongly considered, even in the setting of an unresectable primary NET tumor.

According to the European Neuroendocrine Tumor (ENET) system, as well as other investigators, the presence of extrahepatic metastatic disease is a strong negative prognostic factor and should be considered a relative contraindication to surgery among patients with NELM. ${ }^{8,9,23,24}$ In the current study, patients with extrahepatic metastasis who underwent resection of NELM had a more than twofold increased risk of death long-term.
Interestingly, the 10-year survival of patients with extrahepatic NET disease who underwent resection of NELM was still almost $40 \%$. Whether patients with non-functional NET who have extrahepatic metastatic disease and extensive NELM benefit from surgery is controversial. Mayo et al. reported on patients with NELM who were treated with surgery versus intra-arterial therapy (IAT) and noted an overall improved survival with surgery ${ }^{23}$ However, among patients who had an NF-NET and large $(>50 \%)$ liver involvement, there was no difference in survival among patients who underwent resection versus IAT. ${ }^{23}$ Similarly, Kennedy et al. reported the benefit of Y-90 microsphere therapy in the treatment of patients with advanced NETs. ${ }^{25}$ Overall, the presence of extrahepatic metastatic disease has been reported to have a varied effect on long-term prognosis relative to the extent of liver disease. $^{12,13,26,27}$ In one study using the Surveillance, Epidemiology, and End Results (SEER) database, Franko et al. examined patients with non-functional PNETs and reported that while distant metastasis was a negative prognostic marker, surgical resection improved the long- 
term outcome of patients with distal metastasis versus nonsurgical treatments. ${ }^{6}$ However, given the limitations of the SEER dataset, this study had no clear classification of what constituted a 'true' distant metastasis. ${ }^{6}$ Collectively, the data would suggest that hepatic resection of NF-NELM may be reasonable in the presence of low-volume extrahepatic disease. ${ }^{8,28}$ However, given the higher risk of poor long-term outcomes defined in the current study, patients with a large burden of intra- and extrahepatic disease may not derive a strong benefit from surgery, and less invasive therapeutic approaches such as IAT should be considered.

The current study had several limitations. The multi-institutional nature of the cohort allowed for an increased sample size to examine a relatively rare disease, yet selection criteria for surgery among different centers may have been inconsistent, and the heterogeneity of the patient population may have allowed for certain biases. In addition, only patients who underwent hepatic resection for NFNELM were included in the current database; therefore, 'control' patients who were untreated or received non-surgical treatment were not available for comparison purposes. As such, the survival benefit of resection versus nonsurgical treatments among patients with presence of primary unresected or extrahepatic metastatic disease might be limited in the current study, which needs to be further evaluated.

\section{CONCLUSIONS}

At the time of surgical treatment for NF-NELM, approximately 1 of 7 and 1 of 10 patients presented with an unresectable primary tumor and/or extrahepatic disease, respectively. Patients with primary unresected NET had a comparable survival versus patients who had the primary NET resected. In contrast, survival among patients with extrahepatic metastatic disease was worse versus patients who had intrahepatic-only disease. While surgery should be considered for patients with NF-NELM who have an unresectable primary tumor, operative resection of NF-NELM may not be as beneficial in patients with extrahepatic disease.

ACKNOWLEDGMENT Jun-Xi Xiang and Xu-Feng Zhang were supported by the Clinical Research Award of the First Affiliated Hospital of Xi' an Jiaotong University of China (No. XJTU1AF-CRF2017-004).

AUTHOR CONTRIBUTIONS Study design: J.X. Xiang, X.F. Zhang, T.M. Pawlik. Data collection and interpretation: E.W. Beal, L. Aldrighetti, G.A. Poultsides, T.W. Bauer, R.C. Fields, S.K. Maithel, H.P. Marques, M. Weiss. Data analysis: J.X. Xiang, X.F. Zhang. Drafting of the manuscript: X.J. Xiang, X.F. Zhang. Revision of the draft version of the manuscript: X.F. Zhang, T.M. Pawlik.

CONFLICTS OF INTEREST None declared.

\section{REFERENCES}

1. Gray KD, Moore MD, Panjwani S, et al. Predicting Survival and Response to Treatment in Gastroesophageal Neuroendocrine Tumors: An Analysis of the National Cancer Database. Ann Surg Oncol. 2018;25:1418-24.

2. Saxena A, Chua TC, Sarkar A, et al. Progression and survival results after radical hepatic metastasectomy of indolent advanced neuroendocrine neoplasms (NENs) supports an aggressive surgical approach. Surgery. 2011;149:209-20.

3. Frilling A, Modlin IM, Kidd M, et al. Recommendations for management of patients with neuroendocrine liver metastases. Lancet Oncol. 2014;15:e8-21.

4. Bilimoria KY, Tomlinson JS, Merkow RP, et al. Clinicopathologic features and treatment trends of pancreatic neuroendocrine tumors: analysis of 9,821 patients. $J$ Gastrointest Surg. 2007;11:1460-7; discussion 7-9.

5. Ellison TA, Wolfgang CL, Shi C, et al. A single institution's 26-year experience with nonfunctional pancreatic neuroendocrine tumors: a validation of current staging systems and a new prognostic nomogram. Ann Surg. 2014;259:204-12.

6. Franko J, Feng W, Yip L, Genovese E, Moser AJ. Non-functional neuroendocrine carcinoma of the pancreas: incidence, tumor biology, and outcomes in 2,158 patients. J Gastrointest Surg. 2010;14:541-8.

7. Kent RB, 3rd, van Heerden JA, Weiland LH. Nonfunctioning islet cell tumors. Ann Surg. 1981;193:185-90.

8. Spolverato G, Bagante F, Aldrighetti L, et al. Management and outcomes of patients with recurrent neuroendocrine liver metastasis after curative surgery: an international multi-institutional analysis. J Surg Oncol. 2017;116:298-306.

9. Zhang XF, Beal EW, Weiss M, et al. Timing of disease occurrence and hepatic resection on long-term outcome of patients with neuroendocrine liver metastasis. $J$ Surg Oncol. 2018;117:171-81.

10. Rindi G, Kloppel G, Alhman H, et al. TNM staging of foregut (neuro)endocrine tumors: a consensus proposal including a grading system. Virchows Arch. 2006;449:395-401.

11. Jin K, Xu J, Chen J, et al. Surgical management for non-functional pancreatic neuroendocrine neoplasms with synchronous liver metastasis: a consensus from the Chinese Study Group for Neuroendocrine Tumors (CSNET). Int J Oncol. 2016;49:19912000.

12. Farley HA, Pommier RF. Treatment of Neuroendocrine Liver Metastases. Surg Oncol Clin N Am. 2016;25:217-25.

13. Graff-Baker AN, Sauer DA, Pommier SJ, Pommier RF. Expanded criteria for carcinoid liver debulking: Maintaining survival and increasing the number of eligible patients. Surgery. 2014;156:1369-76; discussion 76-7.

14. Klimstra DS, Modlin IR, Coppola D, Lloyd RV, Suster S. The pathologic classification of neuroendocrine tumors: a review of nomenclature, grading, and staging systems. Pancreas. 2010;39:707-12.

15. Spolverato G, Vitale A, Ejaz A, et al. Net health benefit of hepatic resection versus intraarterial therapies for neuroendocrine liver metastases: a Markov decision model. Surgery. 2015;158:339-48.

16. Kulke MH, Shah MH, Benson AB 3rd, et al. Neuroendocrine tumors, version 1.2015. J Natl Compr Canc Netw. 2015;13:78108.

17. Zerbi A, Falconi M, Rindi G, et al. Clinicopathological features of pancreatic endocrine tumors: a prospective multicenter study in Italy of 297 sporadic cases. Am J Gastroenterol. 2010;105:1421-9.

18. Hochwald SN, Zee S, Conlon KC, et al. Prognostic factors in pancreatic endocrine neoplasms: an analysis of 136 cases with a 
proposal for low-grade and intermediate-grade groups. $J$ Clin Oncol. 2002;20:2633-42.

19. Kunz PL, Reidy-Lagunes D, Anthony LB, et al. Consensus guidelines for the management and treatment of neuroendocrine tumors. Pancreas. 2013;42:557-77.

20. Mayo SC, de Jong MC, Bloomston M, et al. Surgery versus intraarterial therapy for neuroendocrine liver metastasis: a multicenter international analysis. Ann Surg Oncol. 2011;18:3657-65.

21. Andres A, Toso C, Adam R, et al. A survival analysis of the liverfirst reversed management of advanced simultaneous colorectal liver metastases: a LiverMetSurvey-based study. Ann Surg. 2012;256:772-8; discussion 8-9.

22. Givi B, Pommier SJ, Thompson AK, Diggs BS, Pommier RF. Operative resection of primary carcinoid neoplasms in patients with liver metastases yields significantly better survival. Surgery. 2006;140:891-7; discussion 7-8.

23. Mayo SC, de Jong MC, Pulitano C, et al. Surgical management of hepatic neuroendocrine tumor metastasis: results from an international multi-institutional analysis. Ann Surg Oncol. 2010;17:3129-36.
24. Ejaz A, Reames BN, Maithel S, et al. The impact of extrahepatic disease among patients undergoing liver-directed therapy for neuroendocrine liver metastasis. J Surg Oncol. 2017;116:841-7.

25. Kennedy AS, Dezarn WA, McNeillie P, et al. Radioembolization for unresectable neuroendocrine hepatic metastases using resin 90Y-microspheres: early results in 148 patients. Am J Clin Oncol. 2008;31:271-9.

26. Chambers AJ, Pasieka JL, Dixon E, Rorstad O. The palliative benefit of aggressive surgical intervention for both hepatic and mesenteric metastases from neuroendocrine tumors. Surgery. 2008;144:645-51; discussion 51-3.

27. Boudreaux JP, Putty B, Frey DJ, et al. Surgical treatment of advanced-stage carcinoid tumors: lessons learned. Ann Surg. 2005;241:839-45; discussion 45-6.

28. Mayo SC, de Jong MC, Pawlik TM. Surgical management and emerging therapies to prolong survival in metastatic neuroendocrine cancer. Ann Surg Oncol. 2011;18 Suppl 3:S220-1; author reply $\mathrm{S} 2-3$. 